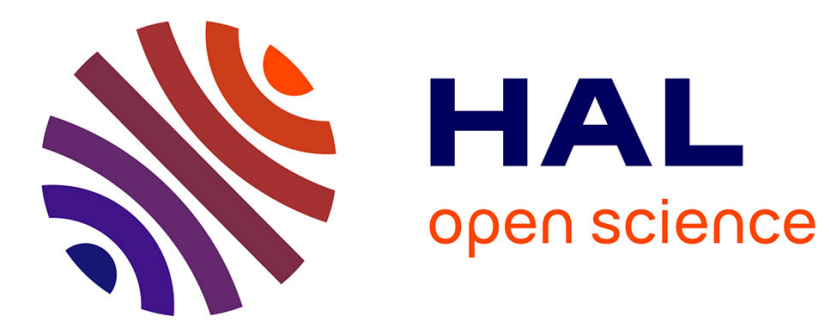

\title{
Condition Monitoring of a Friction Drive System
}

Diego Jair Rodriguez Obando, John Jairo Martinez Molina, Christophe

Bérenguer

\section{To cite this version:}

Diego Jair Rodriguez Obando, John Jairo Martinez Molina, Christophe Bérenguer. Condition Monitoring of a Friction Drive System. CCAC 2017 - 3rd IEEE Colombian Conference on Automatic Control, Oct 2017, Cartagena de Indias, Colombia. 10.1109/CCAC.2017.8276461 . hal-01628575

\section{HAL Id: hal-01628575 \\ https://hal.science/hal-01628575}

Submitted on 3 Nov 2017

HAL is a multi-disciplinary open access archive for the deposit and dissemination of scientific research documents, whether they are published or not. The documents may come from teaching and research institutions in France or abroad, or from public or private research centers.
L'archive ouverte pluridisciplinaire HAL, est destinée au dépôt et à la diffusion de documents scientifiques de niveau recherche, publiés ou non, émanant des établissements d'enseignement et de recherche français ou étrangers, des laboratoires publics ou privés.

$$
\text { Copyright }
$$




\title{
Condition Monitoring of a Friction Drive System
}

\author{
Diego J. Rodriguez, John J. Martinez, Christophe Berenguer \\ Univ. Grenoble Alpes, CNRS, GIPSA-lab, \\ F-38000 Grenoble, France \\ Email: [diego.rodriguez-obando, john.martinez, christophe.berenguer]@gipsa-lab.fr
}

\begin{abstract}
This paper presents an approach for condition monitoring of a friction drive system, based on a recently developed deterioration estimation approach which includes the mechanical device dynamics and the dynamics of the deterioration at the joint. In this work a fault detection and a fault estimation application are proposed. Here, a fault is considered as an abnormal condition of the joint, mainly linked to the quality of the materials. For these purposes, we assume the knowledge of the input current signal and angular-speed sensor information. An architecture for condition monitoring is illustrated with abrupt fault and incipient fault simulated scenarios.
\end{abstract}

\section{INTRODUCTION}

Friction-based devices are ubiquitous in mechanics; some examples like clutch, belt drive and friction drive are mechanisms that use direct power transfer to the load, instead of a chain and sprockets. Of course, one of the problems of those mechanisms is that this useful friction phenomena causes at the same time the wear of the surfaces in contact. This wear causes, in turn, deterioration and eventually faults or the failure of the actuator. The deterioration of this kind of actuators is unavoidable and in consequence optimal control actions are needed to manage the actuators during their useful lifetime, to get a trade-off between efficiency and maintenance.

In any case, the first step to get the current state of health of the contact is to have a reliable condition monitoring system. In our previous work [1], a minimal robustly positive invariantset (mRPI) are calculated in order to characterize all possible trajectories of the angular speeds, which are used to detect a possible failure of the actuator. As advantage, this approach depends only on the angular speeds analysis; nevertheless, it is suggested a more complete on-line analysis of the contact. In [2], an estimator of current state of contact-deterioration in a friction drive is presented. The deterioration is in turn calculated from the estimation of two parameters, namely: a contact quality coefficient $\alpha$, and the parameter of change of this quality with respect to the deterioration $m$. As advantage, this approach gives the estimations with a confidence level. In both approaches, it is suggested the use in fault detectionestimation applications, to use them in online decision making activities such as fault mitigation, mission re-planing and maintenance.

In this work, our main assumption is that the estimator of the parameter $m$, could be used as an appropriate indicator of the changes in the materials in contact, considering the error between the estimates of the parameter $m$ and its nominal values, and other technical restrictions. These changes of materials can be understood as faults under a predefined operation framework. Additionally, another assumption is that the approach can estimate abrupt and incipient faults. Therefore, a method of condition monitoring of a friction drive is explained, based on the analysis of fault detection-estimation scenarios.

The paper is organized as follows: Section II summarizes the description of the system. Section III presents the definitions of deterioration and faults under the framework of the friction drive, and explains an application motivation for this paper. Section IV shows the design of an observer for the parameters $\alpha$ and $m$ of the friction drive. Section $\mathrm{V}$ explains evaluates an architecture for condition monitoring by means of simulated scenarios. Finally, conclusions are given in VI.

\section{DESCRIPTION OF ROLLER-ON-TIRE SYSTEM}

The considered system shown in Fig.1 is called roller-ontire actuator. The nomenclature is shown in Table I. This is a friction drive system composed by a driver device (a dc motor) and a driven device (a wheel). The motor drives the wheel acting directly over the tire. This actuator is modeled as an Uncertain Linear System in the previous work [1]. As depicted in Fig. 1, both devices are affected by the contact force $F_{c}$. It is produced by the motor and causes a torque which drives the wheel and depends on the tangential speeds produced for both motor and wheel, denoted as $v_{1}$ and $v_{2}$ respectively. Therefore, the assumption in the model is that $F_{c}(t)$ is proportional to the relative tangential speed at the contact level, denoted $\Delta_{v}$. That is, $F_{c}(t)=\alpha \Delta_{v}=\alpha\left(r_{1} \omega_{1}-r_{2} \omega_{2}\right)$, where $\Delta_{v}=v_{1}(t)-v_{2}(t)$ and $\alpha \geq 0$ is an uncertain parameter, denoted in the context of our work as the contact quality coefficient. The parameter $\alpha$ characterizes the quality of the contact, e.g. the inter-surface adhesion and the surface roughness, between both rotational devices.

$F_{c}(t)$ can be characterized by using dynamic friction models, see for instance [3] and [4]. Due to the fact that in this work we focus the analysis for long periods of time, only macroscopic friction phenomena are kept to develop a simpler contact model.

Using Newton's laws of motion, the roller-on-tire dynamics are written in the state space representation:

$$
\begin{aligned}
& \dot{x}=A(\alpha) x+B u \\
& y=C x
\end{aligned}
$$

where $x:=\left[\omega_{1}(t) \omega_{2}(t)\right]^{T}$ is the system state, $u=I(t)$ is the control input (in this case, the electrical motor current) and $\alpha$ stands for the uncertain parameter (the scheduling parameter 


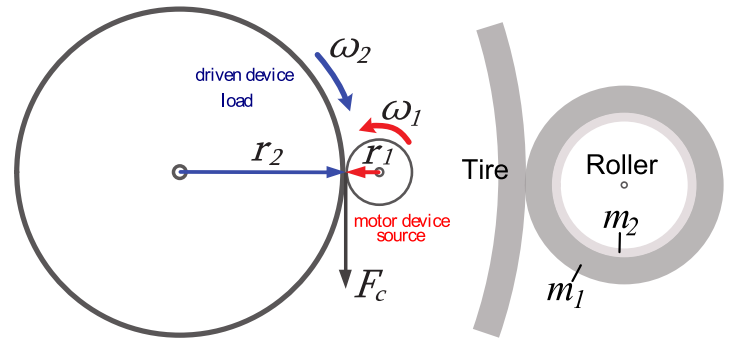

Fig. 1. At left, the roller-on-tire system. At rigth, a zoom of the contact.

TABLE I

NOMENCLATURE

\begin{tabular}{|c|c|c|c|}
\hline Symb. & Value & Units & Physical meaning \\
\hline$v_{1}$ & & {$[\mathrm{~m} / \mathrm{s}]$} & Tangential speed of the motor \\
\hline$v_{2}$ & & {$[\mathrm{~m} / \mathrm{s}]$} & Tangential speed of the driven device \\
\hline$\omega_{1}$ & & {$[\mathrm{rad} / \mathrm{s}]$} & Angular speed of the motor \\
\hline$\omega_{2}$ & & {$[\mathrm{rad} / \mathrm{s}]$} & Angular speed of the driven device \\
\hline$\dot{\omega}_{1}$ & & {$\left[\mathrm{rad} / \mathrm{s}^{2}\right]$} & Angular acceleration of the motor \\
\hline$\dot{\omega}_{2}$ & & {$\left[\mathrm{rad} / \mathrm{s}^{2}\right]$} & Angular acceleration of the driven device \\
\hline$I$ & & {$[A]$} & Electrical motor current \\
\hline$r_{1}$ & 0.0315 & {$[m]$} & External radius of the motor \\
\hline$r_{2}$ & 0.35 & {$[m]$} & External radius of the driven the device \\
\hline$B_{1}$ & $6.36 \times 10^{-3}$ & {$\left[\mathrm{Kgm}^{2} / \mathrm{s}\right]$} & Viscous damping coefficient of the motor \\
\hline$B_{2}$ & $1.76 \times 10^{-3}$ & {$\left[\mathrm{Kgm}^{2} / \mathrm{s}\right]$} & Viscous damping coefficient of the driven device \\
\hline$J_{1}$ & $3.47 \times 10^{-4}$ & {$\left[\mathrm{Kgm}^{2}\right]$} & Moment of inertia of the motor \\
\hline$J_{2}$ & 0.2 & {$\left[\mathrm{Kgm}^{2}\right]$} & Moment of inertia of the driven device \\
\hline$K_{m}$ & 0.0477465 & 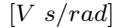 & Motor back-electromotive force constant \\
\hline$\alpha(0)$ & 10 & {$[N / m]$} & Contact quality coefficient \\
\hline$m$ & 0.01 & - & Nominal value of the rate of change of $\alpha$ \\
\hline
\end{tabular}

in the case of a linear parameter varying model interpretation), with matrices:

$$
\begin{gathered}
A(\alpha)=\left[\begin{array}{cc}
\left(-\alpha r_{1}^{2}-B_{1}\right) / J_{1} & \alpha r_{1} r_{2} / J_{1} \\
\alpha r_{2} r_{1} / J_{2} & \left(-\alpha r_{2}^{2}-B_{2}\right) / J_{2}
\end{array}\right], \\
B=\left[\begin{array}{c}
K_{m} / J_{1} \\
0
\end{array}\right]
\end{gathered}
$$

and $C$ an identity matrix, that means that both: angular speed of the motor and angular speed of the driven device are measured, i.e. $y=\left[\omega_{1}(t) \omega_{2}(t)\right]^{T}$.

\section{Deterioration-BASED FAUlt DETECTION}

\section{A. Definition of deterioration for the roller-on-tire system}

The deterioration is defined here as a measure of the loss in the actuator ability to transfer power to the load device. In [1], a dissipation-energy based model of deterioration is presented. There, the dissipated energy is considered as an image of the heat and the material worn at the contact level during traction. This assumption is very similar to the Archard's equation that is more commonly used in railway industry for wear prediction (see [5] and [6]). Thus, a deterioration index is obtained as:

$$
D(t):=\int_{0}^{t} P_{c}(t) d t=\int_{0}^{t} \alpha\left(r_{1} \omega_{1}-r_{2} \omega_{2}\right)^{2} d t
$$

where $P_{c}(t)$ is the dissipated power at the contact level.

In the model we consider that the contact quality coefficient $\alpha$ decreases monotonically in time, for modeling the deterioration. By assumption, the parameter $\alpha(t)$ decreases as $D(t)$ increases. Thus, a first order linear variation of $\alpha$ with respect to $D$, with initial value $\alpha(0)>0$, is defined:

$$
\alpha(t)=-m D(t)+\alpha(0)
$$

where $m$ and $\alpha(0) \in \mathbb{R}^{+}$, and are considered as unknown parameters, but belonging to a given known interval. The parameter $m$, is the rate of variation of $\alpha$ with respect to the deterioration $D$. Therefore, using (5) and (6) we can compute the dynamics of the parameter $\alpha(t)$, as follows:

$$
\dot{\alpha}(t)=-m p(x) \alpha(t)
$$

where the sliding factor $p(x) \geq 0$, is given by $p(x):=\left(r_{1} \omega_{1}-\right.$ $\left.r_{2} \omega_{2}\right)^{2}=\Delta_{v}^{2}$.

From (6) we obtain the normalized deterioration, defined as $\bar{D}(t):=(m / \alpha(0)) D(t)$, where $0 \leq \bar{D}(t) \leq 1$. Thus, for a given initial condition $\alpha(0), \bar{D}(t)$ can be computed at every time-instant using $\alpha(t)$ :

$$
\bar{D}(t)=1-\frac{\alpha(t)}{\alpha(0)}
$$

The deterioration $\bar{D}(t)$ tends to 1 as the quality coefficient $\alpha(t)$ tends to 0 . This normalized deterioration has the advantage to depend only on $\alpha(t)$ and $\alpha(0)$. The latter point requires the knowledge of the possible evolution of the contact quality coefficient $\alpha(t)$. This can be possible by using the dynamics (7) but it requires an estimation of the current and possible futures values of the parameter $m$.

\section{B. Definition of fault in the friction drive}

We focus our interest in the parameter $m$, due to it represents changes in the time-derivative of the quality of the contact $\alpha$. These changes could depend on the material properties and are not produced by operational conditions modeled by the function $p(x)$. Hence, all variations of $m$ beyond admissible thresholds are considered here as faults of the actuator.

The problem in this case is to estimate the variations of $m$, including the model and measurements uncertainties, to detect and possibly estimate faults in the friction drive. A suitable non-linear state-observer is proposed in [2] for the estimation of $\alpha$ and $m$. This is addressed in Section IV under the framework of the present article. From now on, dependence on $t$ is not written sometimes explicitly for easing the notation.

\section{A possible mechanical configuration}

Fig. 1 also illustrates a novel configuration of the materials at the joint of the friction drive, which is part of the motivation for this work. Here, the driver device of the friction drive has two concentric surfaces; in turn, their contact with the tire are characterized by the parameter $m_{1}$ and $m_{2}$. This configuration could allow the operation of the friction drive until the full deterioration of the material with $m_{1}$ after which, the friction drive begins to wear the material with $m_{2}$. We assume both materials and the one of the tire, can be designed previously under laboratory conditions with predefined $m_{1}$ and $m_{2}$. 


\section{ON THE ESTIMATION OF $\alpha$ AND $m$}

This section follows the analysis given in [2], according to the posed problem in this article. Consider the augmented system, using (1)-(2) and (7).

$$
\begin{aligned}
\dot{x} & =A(\alpha) x+B u \\
\dot{\alpha} & =-m p(x) \alpha \\
\dot{m} & =0
\end{aligned}
$$

\section{A. Observability properties of the system}

Considering that parameter $\alpha$ affects the matrix $A(\alpha)$ in an affine way in (3) and the availability of measurements $y=x$, the estimation of the state $\alpha$ can be possible. The estimation of $\alpha$ surely requires enought degree of variation concerning $y$ and $u$. The latter follows the notion of Persistence of Excitation, see for instance [7]. Thus, the electrical current $u=I(t)$ has to be different from zero and suitably varying in time to increase the observability of the state $\alpha$ in the nonlinear system (9)(11).

In terms of the observability of the parameter $m$, remark that it also appears into the dynamical equation characterizing the evolution of $\alpha$ in (10). There, the variation of the parameter $\alpha$ depends on the parameter $m$ in an affine way. Thus, this parameter can be also estimated using previous estimations of $\alpha$ and its time-derivative. As a consequence, the estimation of $m$ requires persistence of the excitation on $\alpha$. In other words, it is necessary to deteriorate the system to improve the estimations.

Due this nonlinear system is observable, it is possible to design an Extended Kalman Filter to estimate the states $x$, and the parameters $\alpha$ and $m$, by considering the knowledge of the input $u=I(t)$ and the available signals $\omega_{1}(t)$ and $\omega_{2}(t)$.

\section{B. Synthesis of an Extended Kalman Filter}

Defining the vector state of the augmented system as $\boldsymbol{x}:=\left[\begin{array}{llll}\omega_{1}(t) & \omega_{2}(t) & \alpha(t) & m\end{array}\right]^{\top}$, the control input $u=I(t)$, and assuming that at every time instant $\omega_{1}(t)$ and $\omega_{2}(t)$ are available from the sensors, the state transition and the system output in continuous time are respectively:

$$
\begin{aligned}
& \dot{\boldsymbol{x}}=f(\boldsymbol{x})+B u+\boldsymbol{w} \\
& \boldsymbol{y}=\boldsymbol{C} \boldsymbol{x}+\boldsymbol{v}
\end{aligned}
$$

with

$$
\boldsymbol{C}=\left[\begin{array}{llll}
1 & 0 & 0 & 0 \\
0 & 1 & 0 & 0
\end{array}\right]
$$

and where $\boldsymbol{w}$ represent the process noise and $\boldsymbol{v}$ the measurement noise, which are both assumed to be Gaussian noises with zero mean and covariance $Q$ and $R$ respectively.

The following covariance matrices are selected:

$$
Q=\operatorname{diag}\left(\left[\begin{array}{cccc}
0 & 0 & 0 & \sigma_{m}^{2}
\end{array}\right]\right) ; \quad R=\operatorname{diag}\left(\left[\begin{array}{ll}
\sigma_{1}^{2} & \sigma_{2}^{2}
\end{array}\right]\right)
$$

where $\sigma_{m}^{2}$ stands for the disturbance variance affecting the behavior of the state $m$. The symbols $\sigma_{1}^{2}$ and $\sigma_{2}^{2}$ represent the sensor noise variances in speed sensors measurements $\omega_{1}$ and $\omega_{2}$, respectively.

The chosen matrix $Q$ takes into account the fact that in the model (9)-(11) the state $m$ can be affected by neglected and/or unmodelled dynamics. That means that we accept the model can be far from the real process, but this model error is only associated to the lack of knowledge on the behavior of $m$. On the other hand, the matrix $R$ considers that both sensors are affected by the same level of measurement noise, and these level noises are relatively smaller than possible state disturbances and/or model errors.

The estimation process is performed as follows: assuming the availability of discrete-time measurements at every timeinstant, with a sample time $t_{s}$, the a priori prediction of the state estimate can be calculated using the continuous-time state transition model:

$$
\dot{\hat{\boldsymbol{x}}}_{k \mid k-1}=f\left(\hat{\boldsymbol{x}}_{k-1 \mid k-1}\right)+B u_{k-1}
$$

and the estimated output: $\hat{\boldsymbol{y}}_{k \mid k-1}=\boldsymbol{C} \hat{\boldsymbol{x}}_{k \mid k-1}$

The prediction of the a priori covariance estimate matrix $\boldsymbol{P}$ is calculated at every time instant as:

$$
\boldsymbol{P}_{k \mid k-1}=\boldsymbol{F}_{k-1} \boldsymbol{P}_{k-1 \mid k-1} \boldsymbol{F}_{k-1}^{\top}+Q
$$

where $\boldsymbol{F}_{k-1}$ is the Jacobian of the function $f(\boldsymbol{x})$ in discrete time. That is, $\boldsymbol{F}_{k-1}=\exp \left(F t_{s}\right)$ with

$$
F=\left.\frac{\partial f(\boldsymbol{x})}{\partial \boldsymbol{x}}\right|_{\hat{\boldsymbol{x}}_{k \mid k-1}}
$$

the Jacobian of the function $f(\boldsymbol{x})$ in continuous time, calculated as:

$$
\frac{\partial f(\boldsymbol{x})}{\partial \boldsymbol{x}}=\left[\begin{array}{cccc}
F_{11} & F_{12} & F_{13} & 0 \\
F_{21} & F_{22} & F_{23} & 0 \\
F_{31} & F_{32} & F_{33} & F_{34} \\
0 & 0 & 0 & 0
\end{array}\right]
$$

where $F_{11}=-\left(\alpha r_{1}^{2}+B_{1}\right) / J_{1}, F_{12}=\left(\alpha r_{1} r_{2}\right) / J_{1}, F_{13}=$ $\left(r_{1} r_{2} w 2-r_{1}^{2} w_{1}\right) / J_{1}, F_{21}=\left(\alpha r_{1} r_{2}\right) / J_{2}, F_{22}=-\left(\alpha r_{2}^{2}+\right.$ $\left.B_{2}\right) / J_{2}, F_{23}=\left(r_{1} r_{2} w_{1}-r_{2}^{2} w_{2}\right) / J_{2}, F_{31}=-2 \alpha m r_{1}\left(r_{1} w_{1}-\right.$ $\left.r_{2} w_{2}\right), F_{32}=2 \alpha m r_{2}\left(r_{1} w_{1}-r_{2} w_{2}\right), F_{33}=-m\left(r_{1} w_{1}-\right.$ $\left.r_{2} w_{2}\right)^{2}$, and $F_{34}=-\alpha\left(r_{1} w_{1}-r_{2} w_{2}\right)^{2}$.

The innovation covariance, denoted $\boldsymbol{S}_{k}$, is:

$$
\boldsymbol{S}_{k}=\boldsymbol{C P}_{k \mid k-1} \boldsymbol{C}^{\top}+R
$$

and the Kalman gain:

$$
\boldsymbol{K}_{k}=\boldsymbol{P}_{k \mid k-1} \boldsymbol{C}^{\top} \boldsymbol{S}_{k}^{-1}
$$

Considering the prediction error: $\tilde{\boldsymbol{e}}_{k}=\boldsymbol{y}_{k}-\boldsymbol{C} \hat{\boldsymbol{x}}_{k \mid k-1}$ (the innovation), the updating of the state estimate is calculated as $\hat{\boldsymbol{x}}_{k \mid k}=\hat{\boldsymbol{x}}_{k \mid k-1}+\boldsymbol{K}_{k} \tilde{\boldsymbol{e}}_{k}$.

Finally, the a posteriori covariance matrix can be updated with $\boldsymbol{P}_{k \mid k}=\left(\boldsymbol{I}-\boldsymbol{K}_{k} \boldsymbol{C}\right) \boldsymbol{P}_{k \mid k-1}$.

Then, the estimation process re-starts again, taking into account all the updated and estimated state vectors and the covariance matrices. The estimation process requires the initialization of the estimated state at instant $k=0$, and an initial a priori covariance matrix $\boldsymbol{P}_{0 \mid 0}$. 


\section{Stochastic bounds for the state estimation}

Due that in this case the estimation error presents a normal distribution, it is possible to bound the estimation error with a given probability, as follows:

$$
\operatorname{Pr}\left(\tilde{\boldsymbol{x}}_{k \mid k}^{T} \boldsymbol{P}_{k \mid k}^{-1} \tilde{\boldsymbol{x}}_{k \mid k} \leq \boldsymbol{c}^{2}\right)=\operatorname{erf}\left(\frac{\boldsymbol{c}}{\sqrt{2}}\right)
$$

where $\operatorname{erf}(\cdot)$ corresponds to the Gauss error function.

Even if there is a probability that some trajectories of the estimation error $\tilde{\boldsymbol{x}}_{k \mid k}$ go out this set, we can use this set to establish an interval of possible values of the state $\boldsymbol{x}_{k}$ with a given probability. Using geometrical properties of the ellipsoids, bounds on the estimation error $\tilde{\boldsymbol{x}}_{k \mid k}$, denoted $\overline{\boldsymbol{x}}_{k}$, can be obtained as:

$$
\overline{\boldsymbol{x}}_{k}=\operatorname{diag}\left(\boldsymbol{P}_{k \mid k}^{1 / 2}\right) \boldsymbol{c}
$$

In particular, for the element corresponding to the estimation of the parameters $\alpha$ and $m$ we have:

$$
\begin{aligned}
& \hat{\boldsymbol{x}}_{k \mid k}(3)-\overline{\boldsymbol{x}}_{k}(3) \leq \alpha_{k} \leq \hat{\boldsymbol{x}}_{k \mid k}(3)+\overline{\boldsymbol{x}}_{k}(3) \\
& \hat{\boldsymbol{x}}_{k \mid k}(4)-\overline{\boldsymbol{x}}_{k}(4) \leq m_{k} \leq \hat{\boldsymbol{x}}_{k \mid k}(4)+\overline{\boldsymbol{x}}_{k}(4)
\end{aligned}
$$

with a probability equal to $\operatorname{erf}(c / \sqrt{2})$ for normal probability distributions. That means that with $c=3$ we can expect that the real value is within the interval given by the estimates with a probability higher than $99.7 \%$.

\section{Checking the consistence of the innovations}

In practice we can not measure the performance of the observer with respect to the state error measures, since we do not know the true state values. Hence, we can check if the observer is performing correctly in terms of the innovation. It is known that if the observer is working correctly then it shows zero mean and white $\tilde{\boldsymbol{e}}_{k}$ with a covariance $\boldsymbol{S}_{k}$. Thus, we can verify that the observer is consistent by applying the following two procedures: i) check that the innovations are consistent with their covariance and ii) check that the innovations are unbiased and white noise. The first test can be performed by using the following bounds on the innovation signal:

$$
\overline{\boldsymbol{e}}_{k}=\operatorname{diag}\left(\boldsymbol{S}_{k \mid k}^{1 / 2}\right) \boldsymbol{c}
$$

where $c>0$ can be chosen to guarantee that the innovations will be bounded by the above values with a given probability.

If those tests are not verified, it is possible that there exist an under-estimate or an over-estimate of the chosen variances of the disturbances. Thus, the chosen matrices $Q$ and $R$ have to be reformulated or adapted.

\section{USING THE OBSERVER FOR CONDITION MONITORING: A NUMERICAL EXPERIMENT}

\section{A. Predefined operating conditions.}

In this case the observer is tested in scenarios with known and predefined operating conditions, to validate in simulation the quality of the obtained estimations of the parameters $\alpha$ and $m$ with known variations of the input $I(t)$. If the quality of those estimations are technically defined as acceptable, the estimation of current condition of the contact is validated for fault detection and/or fault estimation approaches. Here the used input signal $I(t)$ is a square wave with a predefined amplitude $(20 A)$ and predefined values of duty cycle (period: $2 s$ with the $50 \%$ in this case) and the parameters of the model (9)-(11) are considered as constant parameters. For these scenarios $\alpha(0)=10$ and the nominal $m=0.01$ were used. Table I summarizes the used system parameters.

\section{B. Design for fault detection}

The variations of the parameter $m$ are assumed to be equal to 0 in the augmented system (9)-(11). However, in terms of fault, in a real system changes on $m$ can exist as explained in III. Thus, we focus the design and analysis of the proposed observer for possible variations on $m$ in real applications for which there exist an a priori knowledge about the constitution and behavior of the materials in contact.

Table II summarizes three different assumptions on the possible real dynamics of $m$ in operation, namely: (i) the parameter $m$ is always constant, (ii) the parameter $m$ is piecewise constant, and an abrupt change in the value of $m$ can appears at the instant $k=t^{*}$ (a Dirac delta function models this aspect), and (iii) the parameter $m$ can suffer a progressive change with a rate of change equal to $\varepsilon$ (a possible random but a priori bounded parameter).

TABLE II

POSSIBLE DYNAMICS OF $m$

\begin{tabular}{lll}
\hline Assumption & \multicolumn{1}{c}{ Meaning } \\
\hline$i$ & $\dot{m}=0$ & No fault, $m$ is constant \\
$i i$ & $\dot{m}=\delta\left(t^{*}\right)$ & Abrupt fault - change at $k=t^{*}$ \\
$i i i$ & $\dot{m}=\varepsilon$ & Incipient Fault - progressive change \\
\hline
\end{tabular}

1) Tuning the EKF: In terms of design (tuning of matrices $Q, R$ and $\boldsymbol{P}_{0 \mid 0}$ ), we consider the worst case of fault as the given one by an abrupt change in the parameter $m$. To build a consistent design, we follow the procedure presented in Section IV.

Assuming a known variance of the measurement noises $v$, the matrix $R$ is selected as in (15) with $\sigma_{1}^{2}=1 \times 10^{-8}$ and $\sigma_{2}^{2}=1 \times 10^{-10}$.

Concerning the matrix $Q$ in (15), the chosen value for $\sigma_{m}^{2}$ is obtained by assuming possible abrupt variations on values of $m$. This variations can be modeled as impulse disturbances (a discrete-time Dirac delta), affecting the dynamics of the state $m$ and taking values in the interval $(a, b)=(0.01,0.02)$. If we assume, for instance, that these disturbances are random variables with an uniform probability distribution, for all $k>$ 0 , their variance can be calculated as:

$$
\sigma_{m}^{2}=\operatorname{var}(\delta(k))=\frac{1}{12}(a-b)^{2}
$$

which provides $\sigma_{m}^{2}=8.3333 \times 10^{-6}$.

This value is also used arbitrarily to initialize the covariance matrix as $\boldsymbol{P}_{0 \mid 0}=\operatorname{diag}\left(\left[\begin{array}{llll}\sigma_{m}^{2} & \sigma_{m}^{2} & \sigma_{m}^{2} & \sigma_{m}^{2}\end{array}\right]\right)$.

Additionally, in this numerical example, the Jacobian (19) has been modified by including $F_{44}=-100$. This allows us 

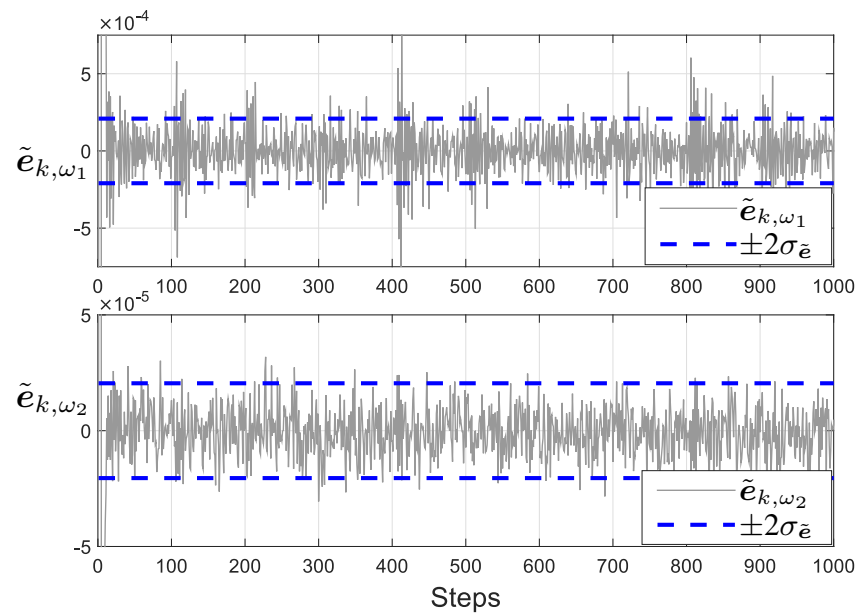

Fig. 2. Prediction output error $\tilde{\boldsymbol{e}}_{k}$ for $\omega_{1}$ and $\omega_{2}$.

to consider a band-pass filter model for $m$ which reduces the effect of noise during the estimation of this variable. That is, the Extended Kalman filter considers the following dynamics $\dot{m}=-100 m$ instead of $\dot{m}=0$ in (11).

2) Analysis of the variance of the innovations: Fig. 2 shows the prediction error $\tilde{\boldsymbol{e}}_{k}$, for both measured signals $\omega_{1}$ and $\omega_{2}$. The dashed lines represent a confidence interval of $\pm 2 \sigma_{\tilde{e}}$ which is computed using (25). As it is shown, the errors $\tilde{\boldsymbol{e}}_{k}$ are zero mean and most of the time they remain inside the confidence interval. Assuming that sensors are affected by Gaussian noise, we can conclude that the estimation error belongs to the chosen confidence interval with a probability greater than $95 \%$.

\section{Analysis of the estimations}

The condition monitoring architecture is shown in Fig.3. The block $F E$ (from fault estimation) compares the current state $\hat{m}$ with nominal-behavior thresholds given by $m_{1}, m_{2} \ldots m_{n}$. This $F E$ could be used also to compare the $\hat{m}$ with a database of $m$ in the sense of a residual generator. The values of the thresholds can be defined as constant levels based on the variance of the estimations. Therefore, this variance can be obtained a priori from the observer, e.g. like $\bar{m}$ (see (23)). This variance can be also obtained experimentally, taking the laboratory-characterization of $m$ for given pairs of material in the tire-roller contact. The boolean indicator $f_{e}$ is set to one every time that $m$ reaches at first time a threshold. In other words, an incipient fault, modeled by a ramp with positive slope $\varepsilon$, is detected when $f_{e}=1$ in different time instants.

Another complementary-detection block $F D$ (from fault detection) detects an unusual behavior including the value of $\alpha$, i.e. using the pair $(m, \alpha)$ as a fault indicator. Fig. 4 outlines a set $\mathcal{S}$ in which there exist the pair $(m, \alpha)$ with safety bounds $\alpha_{-}^{+}$and $m_{-}^{+}$. Every real value of the pair $(m, \alpha)$ is given by (23). Therefore, at every time instant there is a point $(\hat{m}, \hat{\alpha})$ with a confidence level $\pm \bar{m}$ and $\pm \bar{\alpha}$ respectively. Hence, different domains can be identified in the $m, \alpha$ plane corresponding to different possible conditions (e.g.

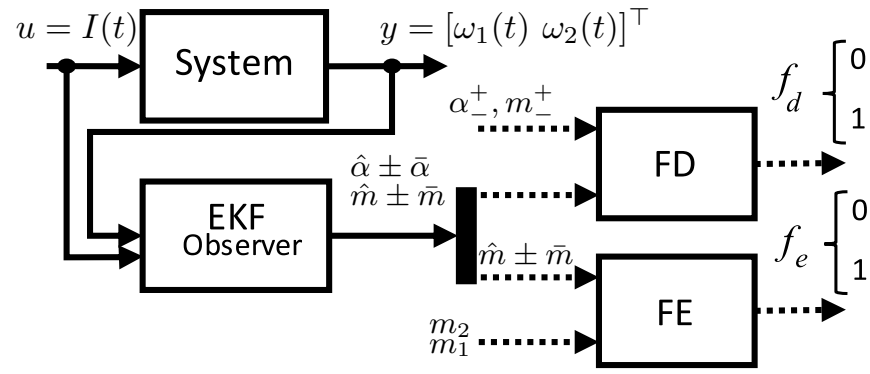

Fig. 3. Condition monitoring for the friction drive based on the estimation of $\alpha$ and $m$.

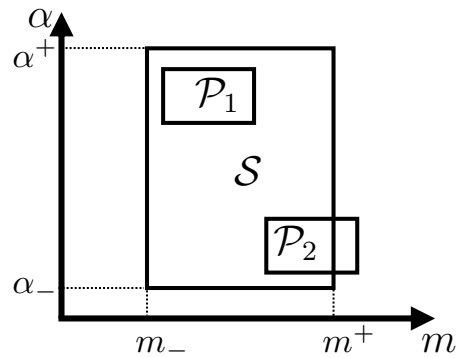

Fig. 4. Set $\mathcal{S}$ in which there exists the pair $(m, \alpha)$.

healthy, faulty, etc.). This is represented by the sets $\mathcal{P}$. In this case $\mathcal{P}_{1}$ represents a healthy condition and $\mathcal{P}_{2}$ represents a faulty condition. The fault indicator is presented as a boolean condition $f_{d}$.

$$
f_{d}=\left\{\begin{array}{l}
0 \text { if } \mathcal{P} \subseteq \mathcal{S} \\
1 \text { otherwise }
\end{array}\right.
$$

Fig. 5 shows the performance of the observer in a scenario with dynamics type (i) on $m$ before $t=10 \mathrm{~s}$. Fig. 5 also shows a dynamics-(ii) scenario, i.e. with an abrupt variation of the nominal value $m$ at $t=10 \mathrm{~s}$. As can be seen, the observer can follow the changes in $m$. Notice also the change in the slope at this time in the curve of $\hat{\alpha}$. The variance of the obtained estimations are consistent with the variances chosen for tuning the Extended Kalman Filter. These results also confirm that the estimations are accurate, as it can be evaluated using the bounds calculated by using (23) and (24). At $t=10 \mathrm{~s}$ the indicator $f_{d}$ becomes 1 . This type of change in $m$ can be understood as a fault or an abrupt material change at the contact level. In consequence, the observer can be useful for robust abrupt-fault detection.

Fig. 6 shows the performance of the observer in a type (iii) scenario, i.e. with a progressive variation of the nominal value $m$ between $t=4 s$ and $t=14 \mathrm{~s}$. As expected, the observer can follow the changes in $m$. The fault indicator $f_{e}=1$, after the two predefined thresholds. Of course, one can set several thresholds with similar results. This means that this approach can be used not only for detecting a fault but also to estimate it or to measure it along the time. In consequence, the observer is useful for robustly detecting incipient faults in the contact properties, given by $m$. 

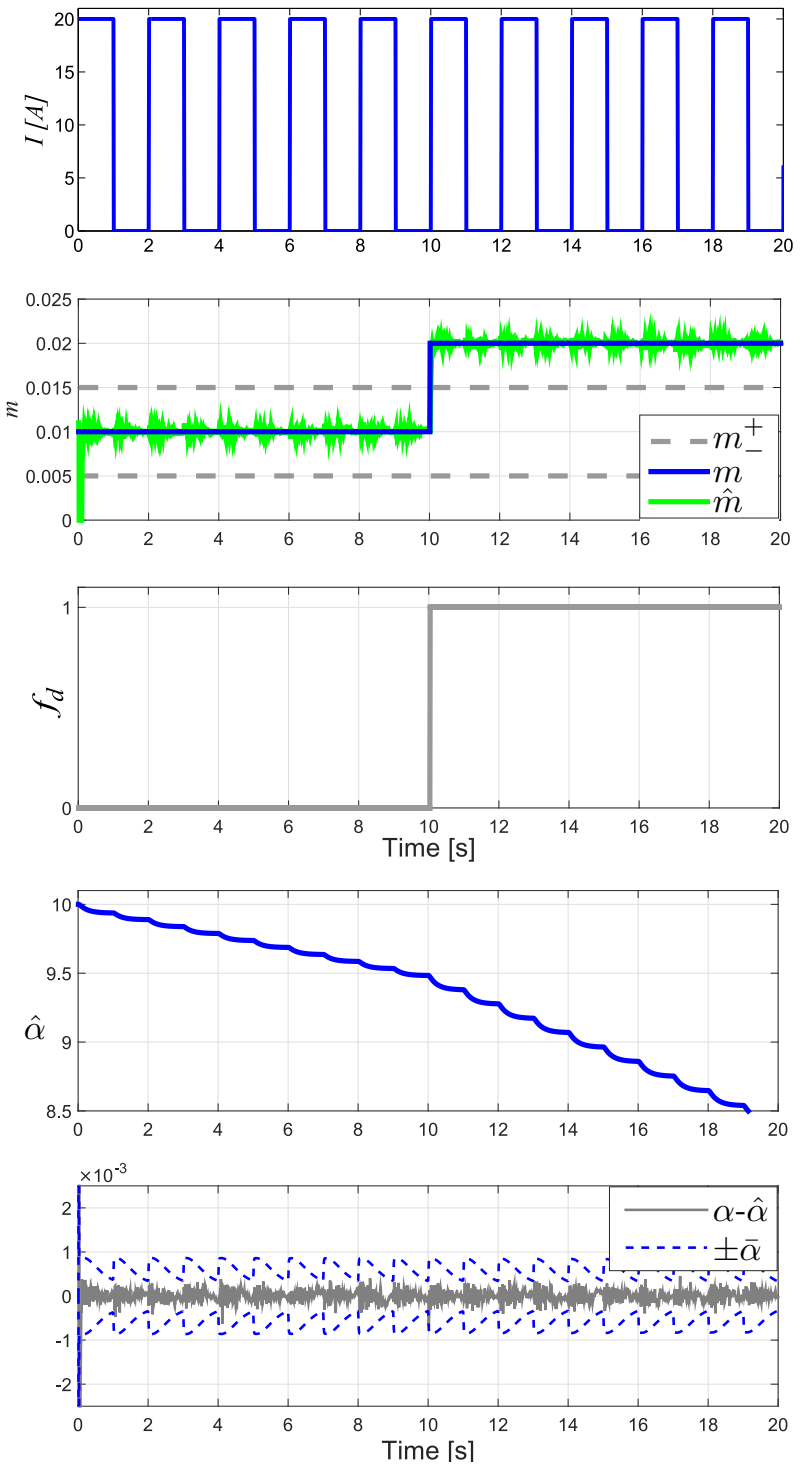

Fig. 5. Input sequence and estimation of the current state of $\hat{m}$ and $\hat{\alpha}$ with an abrupt variation of $m$ at $t=10 \mathrm{~s}$.

Fig. 7 sketches the normalized estimated deterioration $\hat{\bar{D}}$ for the scenario of Fig. 5. As expected, it follows an inverse behavior to $\alpha$. The normalized deterioration $\bar{D}$ is calculated at every time-instant using the estimated value of $\alpha$ and (8).

\section{CONClusions}

This online monitoring approach can detect a fault in the contact power transfer between the both materials in contact in the friction drive, and estimate a fault, giving a measure of the current state of health of the joint of the friction drive. The results show the potential impact of the approach in maintenance applications. This detection-estimation action has the advantage to function within predefined safety zones, which in turn can be used for instance for preventive maintenance actions and to design safer material configurations for friction drives, such as multiple-layer contact materials.
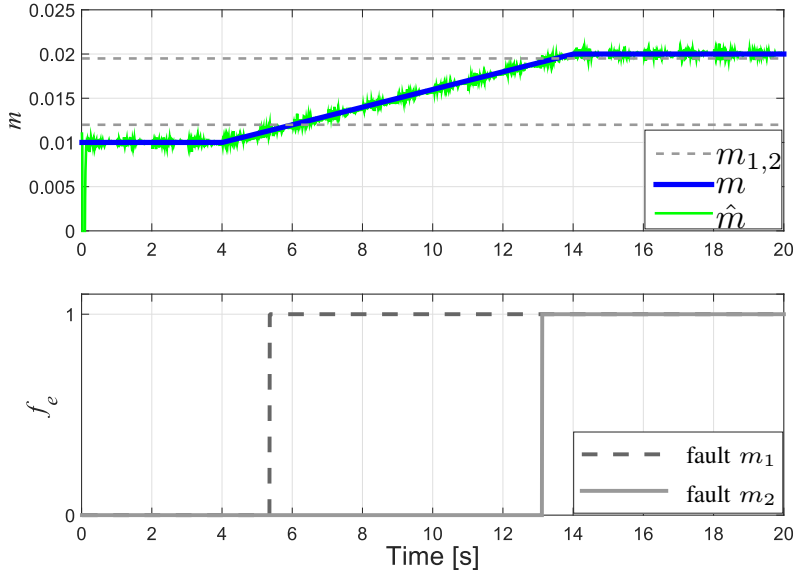

Fig. 6. Estimation of a progressive variation of $m$ and fault detection. The fault in $m_{2}$ corresponds to the case $\mathcal{P}_{2}$ in Fig. 4.

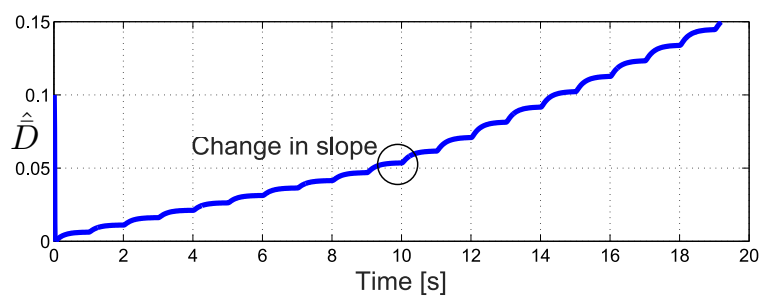

Fig. 7. Condition monitoring of deterioration. Here $\hat{\bar{D}}$ is calculated using (8).

Additionally, changing the material in the contact and assuming all the parameters of the friction drive as constant in multiple laboratory-experiments, the approach could serve to characterize the joint with several pairs of tire-roller material.

\section{ACKNOWLEDGMENT}

This work was partially supported by the French CNRS PEPS 2017 in the context of the IMPROVED project.

\section{REFERENCES}

[1] D. J. Rodriguez Obando, J. J. Martinez Molina, and C. Berenguer, "Set-invariance analysis for deterioration prediction on a roller-on-tire actuator," in Proc. 3rd Conference on Control and Fault-Tolerant Systems - SysTol2016 - 7-9 Sep. 2016 - Barcelona, Spain, 2016, pp. 87-92.

[2] — "Deterioration estimation for remaining useful lifetime prognosis in a friction drive system," in Proc. The 20th World Congress of the International Federation of Automatic Control-IFAC2017 - 9-14 July 2017 - Toulouse, France.

[3] C. Canudas-de Wit, P. Tsiotras, E. Velenis, M. Basset, and G. Gissinger, "Dynamic friction models for road/tire longitudinal interaction," Vehicle System Dynamics, vol. 39, no. 3, pp. 189-226, 2003.

[4] J. Martinez, J. Avila, and C. Canudas-de Wit, "A new bicycle vehicle model with dynamic contact friction," in IFAC Advances in Automotive Control. Salerno, Italy: Elsevier, 2004, pp. 625-630.

[5] A. Bevan, P. Molyneux-Berry, B. Eickhoff, and M. Burstow, "Development and validation of a wheel wear and rolling contact fatigue damage model," Wear, vol. 307, pp. 100-111, 2013.

[6] M. A. Cremona, B. Liu, Y. Hu, S. Bruni, and R. Lewis, "Predicting railway wheel wear under uncertainty of wear coefficient, using universal kriging," Reliability Engineering \& System Safety, vol. 154, pp. 49-59, 2016.

[7] G. Besançon, Nonlinear observers and applications. Springer, 2007, vol. 363 . 